\begin{tabular}{lc}
\hline SCIENCE \& TECHNOLOGY \\
Journal homepage: http://www.pertanika.upm.edu.my/ \\
\hline PERTANIKA
\end{tabular}

\title{
Bifurcation Analysis of an Exothermic Biocatalytic Reaction System
}

\author{
'Afifi Md Desa 1,2*, Mohd Hafiz Mohd' and Mohamad Hekarl Uzir³ \\ ${ }^{1}$ School of Mathematical Sciences, Universiti Sains Malaysia, 11800 USM, Penang, Malaysia \\ ${ }^{2}$ Institute of Engineering Mathematics, Universiti Malaysia Perlis, 02600 UniMAP, Arau, Perlis, Malaysia \\ ${ }^{3}$ School of Chemical Engineering, Universiti Sains Malaysia, Engineering Campus, 14300 USM, Nibong \\ Tebal, Pulau Pinang, Malaysia
}

\begin{abstract}
This paper focuses on the bifurcation analysis of an exothermic biocatalytic reaction system (EBRS). The objectives of the study were to provide a detailed dynamical systems analysis of an exothermic biocatalytic reaction and examine the long-term behaviours of the system using the techniques from phase portraits and bifurcation analysis. We investigated the combined influences of the proportional control constant and dilution rate on the dynamics of the model. Phase portraits relating to distinct outcomes of EBRS were computed to investigate the existence of different attractors in this system and its stability. Under a fixed dilution rate and different values of proportional control constant, there were four distinct outcomes in our model, which were stable steady states, bistability of two stable steady states, bistability between stable steady states and limit cycle and stable limit cycles. A bifurcation analysis (of codimensions one and two) was performed to examine how the overall dynamics change as chemically relevant parameters were varied. We observed that when the values of proportional control constant were high, the system would achieve stable steady states regardless of how fast the dilution rate was. With lower magnitudes of proportional constant control,

ARTICLE INFO

Article history:

Received: 17 September 2020

Accepted: 30 October 2020

Published: 22 January 2021

DOI: https://doi.org/10.47836/pjst.29.1.09

E-mail addresses:

afifimddesa@gmail.com (‘Afifi Md Desa)

mohdhafizmohd@usm.my (Mohd Hafiz Mohd)

chhekarl@usm.my (Mohamad Hekarl Uzir)

* Corresponding author

bistability occurred and the outcomes of this system depended on the initial conditions. Compared to higher dilution rates scenario, reactions that occurred at lower dilution rates provided higher reaction yields for this EBRS operation; it was also observed that both scenarios produced stable steady-state outcomes with different concentrations. In
\end{abstract}


conclusion, the dynamical system analysis of the model improves our understanding of the EBRS under consideration and these insights may be beneficial for optimising operating conditions of this chemical system.

Keywords: Bifurcation, biocatalytic reaction, dynamical systems, nonlinear, phase-plane analysis

\section{INTRODUCTION}

An exothermic reaction occurs when the temperature of a system rises due to the evolution of heat (Petrucci et al., 2002). This is due to the reactant having more energy than the product, which causes the potential excess of energy to change into the kinetic energy of the product formed. During a chemical reaction, a catalyst is introduced to increase the speed of the reaction. A catalyst is an entity that accelerates a chemical reaction without being consumed (Guengerich, 2017). Often catalyst will lower the activation energy of a reaction, and this mechanism can increase the rate of the reaction. Normally, a catalyst does not react with reactants directly but if it does, in the case of enzymatic reaction systems, the intermediate complexes would either remain as bound complexes or they can reversibly dissociate and continue to catalyse the remaining substrate.

Most biocatalysts are protein known as enzymes. Enzymes are a type of biocatalysts and they can be obtained from living cells. Since the first biocatalyst was introduced nearly a century ago, different enzyme-based processes have been commercialised to manufacture several valuable products in many industries (Guan et al., 2011; Hu et al., 2010; Jestin \& Kaminski, 2004; Sekhon et al., 2018; Uhr et al., 2014; Zhao et al., 2018). In contrast to a chemical catalyst, which produces side products, a biocatalyst usually produces a cleaner reaction. As the world is beginning to use green energy, biocatalytic processes will play an important role in the next generation of industrial revolutions for chemical production. The introduction of biocatalysts in chemical reactions can reduce several processing steps which will lead to higher efficiency and time (Tufvesson et al., 2010).

In a chemical reaction involving biocatalyst, the enzymes need to be blended with the reactant for a reaction to occurs. Blending operations are frequently used in many industries to ensure that the final products meet customer requirements. A continuous stirred-tank reactor (CSTR) in shown in Figure 1. The CSTR are commonly used in industrial processing, primarily in homogeneous liquid-phase flow reactions where constant agitation is required. A CSTR is a continuous reactor that is equipped with

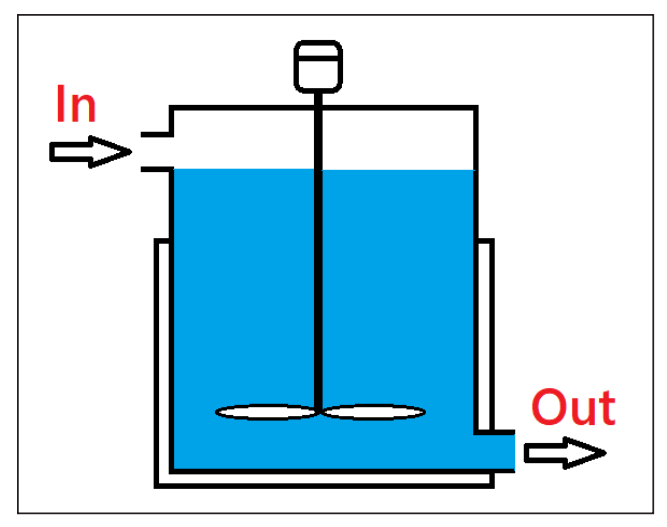

Figure 1. A diagram of a continuous stirred-tank reactor 
an impeller or mixing device for efficient mixing. In a process involving a biocatalyst, a reactant enters the CSTR where the biocatalyst is prepared prior to the reaction and the product formed during the reaction flows through the outlet. There are a few factors influencing the reactor productivity such as substrate and enzyme concentration (Bommarius $\&$ Riebel, 2004), temperature (Devasena, 2010) and the dilution rate or time constant (De Gooijer et al., 1996). Increasing substrate and enzyme concentration will increase the enzyme reaction up to the limit of saturation. However, in practice it is very unlikely to increase to enzyme concentration due to certain constrain such as cost or excess of deactivated protein in the reactor where maximum limit of enzyme concentration is reached. Meanwhile, the effects of temperature on the reactor productivity depends on two factors which is the influence of temperature on the reaction rate and the thermal denaturation of enzyme at elevated temperature (Bommarius \& Riebel, 2004). The vital aspect to achieve higher reactor productivity is to control temperature to the optimum temperature which was one of the focus of this study.

The most complete analysis of a CSTR behaviour was shown in the research by Aris and Amundson (1958a, 1958b). In their work, a simple CSTR model with associated control equipment was formulated to study the transient behaviour of a reaction. Even though many biocatalytic reactions are performed in CSTR, not many models and dynamical systems analyses of this reaction have been carried out in the literature. While there are several modelling studies on biocatalytic reactions, most of these studies focus on the other area than dynamical study including developing model and simulating transient behaviours of the systems (e.g. Petkevicius \& Baronas, 2017), reactor selection for biocatalytic (e.g. Lindeque \& Woodley, 2019), kinetic studies on immobilised enzymes (e.g. Mazzei et al., 2009) and industrial applications (e.g. Choi et al., 2015). One of the studies that explores the dynamics of biocatalytic reactions was conducted by Radzi and Uzir (2009), where they discussed the formulation of the model and the parameters that were involved. The dynamical analysis also covered the following three parameters: proportional control constant, dilution rate and enzyme concentration.

Inspired by the study of Radzi and Uzir (2009), we are interested to further explore the dynamical behaviours of exothermic biocatalytic reaction in CSTR by employing a similar model. One of the objectives of this study was to provide a detailed dynamical systems investigation of an exothermic biocatalytic reaction system (EBRS) using the techniques of phase portraits and bifurcation analysis. Furthermore, we would like to gain a better understanding of the influential roles of dilution rate and proportional control constant in determining the outcomes of this biocatalytic system. This study hopes to provide the researchers and engineers with a better understanding and control of the EBRS and to contribute to the existing body of knowledge in this area.

The article is organised as follows. The model of exothermic biocatalytic reaction is described under the Method section and then it is followed by the phase portrait analysis. We 
illustrate the dynamical outcomes of the system at different values of proportional control constant, $K_{C}$. By using numerical continuation, we discuss some mathematical insight on the effect of proportional control constant and dilution rate on the dynamical outcomes of the system. Finally, we discuss the several CSTR operations implications of our results.

\section{METHODS}

In order to study the biocatalytic reaction, we would examine a dimensionless model of an exothermic biocatalytic reaction, in CSTR which was initially introduced by Radzi and Uzir (2009) (Equation 1).

$$
\begin{aligned}
& \frac{d y}{d \tau}=1-y-\frac{k_{A} y \alpha}{D} e^{\frac{\beta}{\theta}} \\
& \frac{d \theta}{d \tau}=\theta_{0}-\theta+\frac{k_{A} y \alpha}{D} e^{\frac{\beta}{\theta}}-U\left(\theta-\theta_{c}\right)\left[1+K_{C}\left(\theta-\theta_{S}\right)\right]
\end{aligned}
$$

In this model, the rate of change of substrate concentration is given by $\frac{d \theta}{d \tau}$ and the rate of change of the reactor temperature is given by $\frac{d \theta}{d \tau}$. The variable $y$ is a dimensionless unit as it is the concentration of the outflow divided by the concentration of the inflow. If the value of $y$ is close to one, it shows that the CSTR has a higher substrate concentration, and if the value is close to zero it shows that the CSTR has a lower substrate concentration. The term $K_{C}$ is the proportional control constant on the cooling water flow rate. A high value of $K_{C}$ means that the cooling water flow rate is set to a high value. Therefore, the higher cooling water flow rate will reduce the reactor temperature faster. The term $D$ is the dilution rate, which is the rate that the existing medium in the reactor is replaced by a fresh medium. A high value of $D$ means the rate of inflow and outflow from the CSTR is higher. The full interpretations of each variable and parameter values that used in this study were motivated by Radzi and Uzir (2009) and are shown in Table 1.

To achieve the objectives of the study, the first step was to determine the equilibria and their stability by using MAPLE ${ }^{\circledR}$ software. By using Pplane8, the solutions of the system were plotted for different set of parameters and the stability of the equilibria was also established. To gain better insight on the mechanisms that induced distinct solutions in this system for a different sets of parameters, we employed XPPAUT to conduct a codimension-one bifurcation analysis and this analysis tracks the stable and unstable equilibria together with their bifurcation points as parameters were varied. The bifurcation diagrams are important to understand the overall dynamics of this chemical system. Lastly, we also used MATCONT to plot a codimension-two bifurcation diagram to investigate the joint effects of $K_{C}$ and $D$ in determining the dynamics of the system. 
Table 1

The variables and parameters of the model

\begin{tabular}{lll}
\hline $\begin{array}{l}\text { Variable/ } \\
\text { Parameter }\end{array}$ & Description & Value \\
\hline$y$ & Dimensionless substrate concentration & Variable \\
$\theta$ & Dimensionless reactor temperature on the stability of reaction & Variable \\
$\tau$ & Dimensionless time & Variable \\
$k_{A}$ & Reaction velocity constant & $\mathrm{e}^{25}$ \\
$\alpha$ & Dimensionless total enzyme concentration & 60 \\
$D$ & Dilution rate: the rate that the existing medium in the reactor is replaced by & $10 \leq D \leq 90$ \\
& a fresh medium & 50 \\
$\theta_{0}$ & Dimensionless parameter for heat transfer & 1.75 \\
$\theta_{C}$ & Dimensionless initial reactor temperature on the stability of the reaction & 1.75 \\
$\theta_{S}$ & Dimensionless mean temperature of water in the cooling coil & 2 \\
$U$ & Desired steady-state temperature & 1 \\
$K_{C}$ & Dimensionless analogue of $U_{o} A$, the overall heat transfer rate & $1 \leq K_{C} \leq 12$ \\
\hline
\end{tabular}

\section{RESULTS}

In this section, we begin with the phase portrait analysis followed by the codimension-one and -two bifurcation analyses. The phase portrait analysis was performed using Pplane 8 to visualise the equilibria of the system and identify the nature of stability of these solutions (i.e. whether they are stable or unstable). This could be performed for a given set of parameter values to reveal the dynamics of the system. With this analysis, we would be able to locate the nullclines, attractors, repellers and limit cycles for a given set of parameters as in Table 1 and this information should be in agreement with our theoretical analysis, e.g. local stability analysis.

To understand the changes in the dynamics of the system as chemically relevant parameters vary, we conducted a codimension-one bifurcation analysis of the system. This was performed using XPP software and Auto package. Two parameters, which are proportional control constant $\left(K_{C}\right)$ and the dilution rate $(D)$, had been chosen for this analysis as we would like to examine their combined influences on the dynamical behaviours of the system. The proportional control constant $K_{C}$, serves as a heat removal mechanism for the reaction. Since the biocatalytic reaction is an exothermic reaction, heat from the reaction will be released into the surroundings and this process will increase the temperature of the reactor. As the temperature increases, the enzyme activity will be affected because it needs to be operated at their optimal temperature (Devasena, 2010). By examining the influences of $K_{C}$ on the dynamical behaviour of the system, we can control the temperature of the reactor to achieve the optimal operating conditions. On the other hand, the dilution rate, $D$ is the rate at which the existing substrate in the reactor is replaced by a fresh substrate. 
Since an enzyme is being used as a catalyst to speed up the reaction, the reaction will only occur when the substrate binds into the active sites of the enzymes. The duration that the substrate spends in the reactor can shape the efficiency of the reactor.

Bifurcation analysis will also identify the threshold values for $D$ and $K_{C}$ where the change in dynamics occur. We were also able to locate the tipping points where the distinct bifurcations emerged in the system. Further investigation was also carried out using a codimension-two bifurcation analysis to search for different stable regions of the model in a two-parameter space. For this analysis, we relied on MATCONT software to plot these stability regions and examine the interactions of different bifurcations in this CSTR system.

\section{Phase Portrait Analysis}

Since temperature is one of important factors that can influence enzyme activity, we will investigate the effects of changing temperature on the biocatalytic reaction system. This investigation corresponded to varying values of $K_{C}$ to observe the dynamical outcomes of the system under a fixed dilution rate (e.g. $D=50$ ). Our observations are as follows: (i) stable equilibrium; (ii) bistability of two stable steady states; (iii) bistability between the stable equilibrium and limit cycle; and (iv) stable limit cycles. These distinct dynamics are illustrated in Figure 1, which enable us to understand the long-term behaviours of the solution. It is also used to verify the dynamics in the codimension-one and two bifurcation diagrams which is described in a later section.

Figure 1a demonstrates the phase portrait when $K_{C}$ is rather low (i.e., $K_{C}=1$ ). The values of other parameters are listed in Table 1. Figure 1 shows the bistability of the system where there occurred two stable equilibria $\left(E_{1}\right.$ and $\left.E_{3}\right)$ and an unstable saddle point $E_{2}$. At $E_{2}$, the stable manifold of the saddle-type solution (solid black curve) acted as a basin boundary and separated the basin of attractions for the two stable steady states, $E_{1}$ and $E_{3}$. Depending on their initial conditions, the trajectories either converged to $E_{l}$ (lower substrate concentration) or $E_{3}$ (higher substrate concentration). For example, an initial condition below the stable manifold would converge to steady state $E_{3}$. Otherwise, it would converge to a steady state $E_{l}$.

At $K_{C}=2$, the model exhibitedanother type of dynamical behaviour, which was the coexistence of a stable equilibrium with a stable limit cycle (Figure $2 \mathrm{~b}$ ). The previous stable equilibrium in $E_{l}$ (stable spiral) appeared to change a stability and consequently a stable limit cycle emerged with an increase in $K_{C}$; any trajectories that started above the stable manifold of $E_{2}$ (solid black curve) would be attracted to this limit cycle. The occurrence of the limit cycle in this system indicates that an increase in heat removal would cause the stable equilibrium of $E_{1}$ (with high substrate concentration) to lose its stability and this also leads the appearance of a stable limit cycle. 


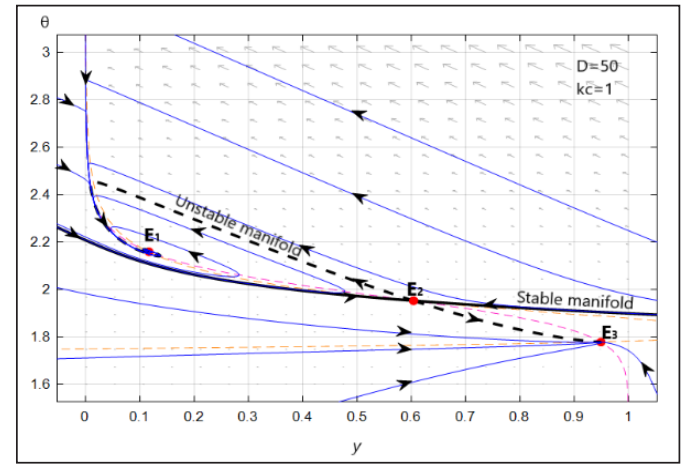

(a)

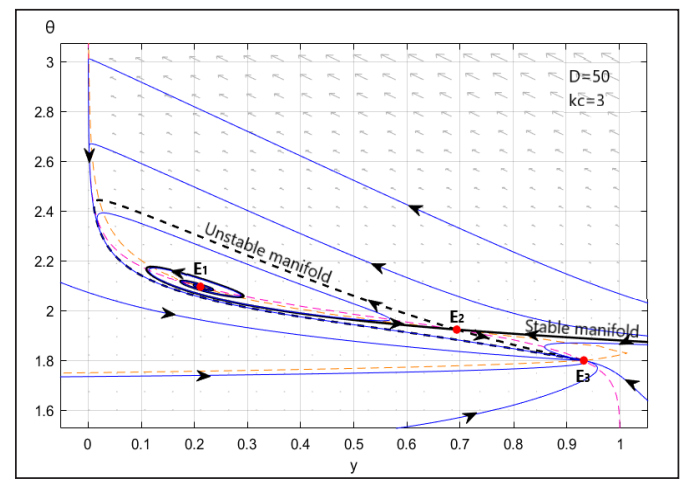

(c)

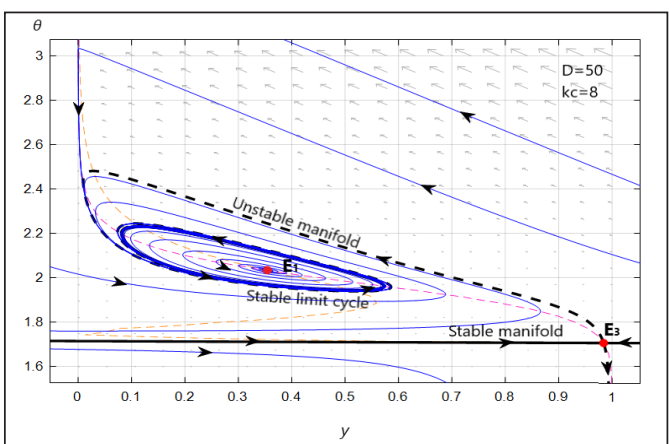

(e)

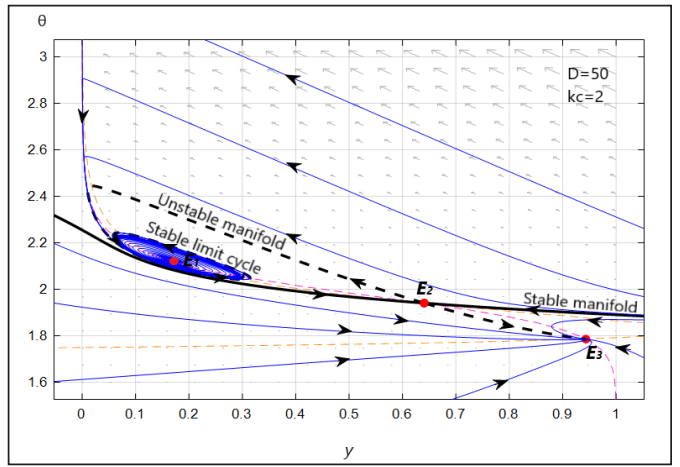

(b)

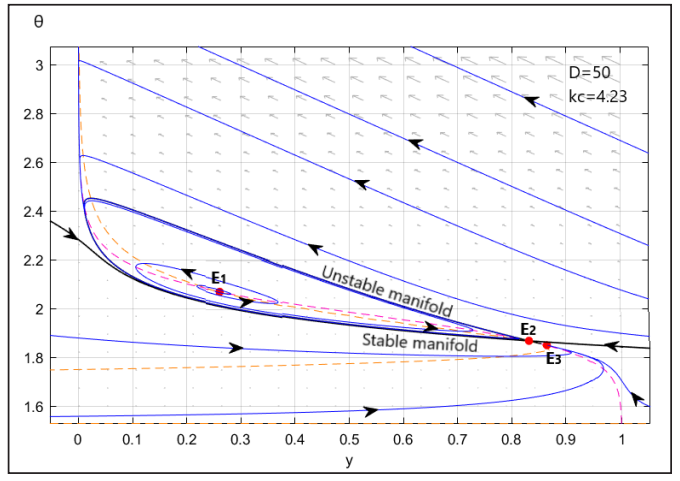

(d)

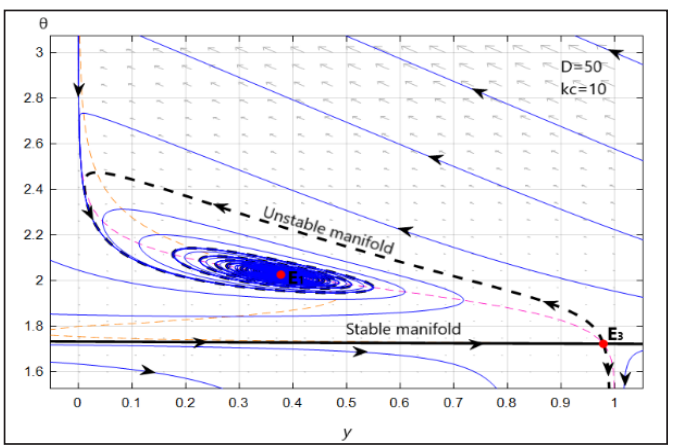

(f)

Figure 2. Phase portrait of the system at $D=50$ and (a) $K_{C}=1$, (b) $K_{C}=2$, (c) $K_{C}=3$, (d) $K_{C}=4.5$, (e) $K_{C}=$ 8 and (d) $K_{C}=10$

At $K_{C}=3$, the previous stable limit cycles could vanish and we observed the emergence of an unstable spiral at $E_{1}$ and hence due to this reason, the bistability phenomenon that we was previously also disappears. This situation results in the occurrence of single stable attractor $E_{3}$ (Figure 2c). In this case, all trajectories would converge to stable equilibrium $E_{3}$.

There was another change in the dynamical behaviour of the model that we realised when the value of $K_{C}$ increase e.g., $K_{C}=4.23$ as in Figure 2d. The unstable spiral previously 
at $E_{1}$ becoming stable limit cycle and hence the bistability phenomenon occurred where stable equilibrium at $E_{3}$ and stable limit cycle at $E_{1}$ coexisted. All trajectories above the stable manifold would be attracted to the limit cycle while trajectories below stable manifold would be attracted to stable equilibrium.

At higher values of $K_{C}$ e.g., $K_{C}=8$ (Figure 2e), we observed that the limit cycle decreased in size, which indicated that the amplitude of oscillation is getting smaller. Figure 3 shows the amplitude of oscillation in $y$ when $K_{C}=8$ had decreased compared to the amplitude at $K_{C}=4.5$. This observation indicates that when $K_{C}$ increases, this situation will reduce the amplitude of oscillatory solution and demonstrate that the conversion of reactant is more efficient. The biocalatytic reaction activity is increased due to the fact that more heat being released from the reactor. The reactor temperature becomes optimal for a biocatalytic process to occurs.

At higher values of $K_{C}$ for instance, $K_{C}=10$, the limit cycle disappeared, and the dynamics were replaced by a stable equilibrium (stable spiral) at $E_{l}$ is still a single attractor in the system, and for the system to reach this steady state the initial conditions must start above the stable manifold (Figure 2f). The system oscillated until it reached the steady state.

\section{Codimension-One Bifurcation Analysis}

To investigate the mechanism behind the emergence and disappearance of certain dynamic in this model, we employed codimension-one bifurcation analysis to track steady states of the model as parameter change. We were conducting the codimension-one bifurcation

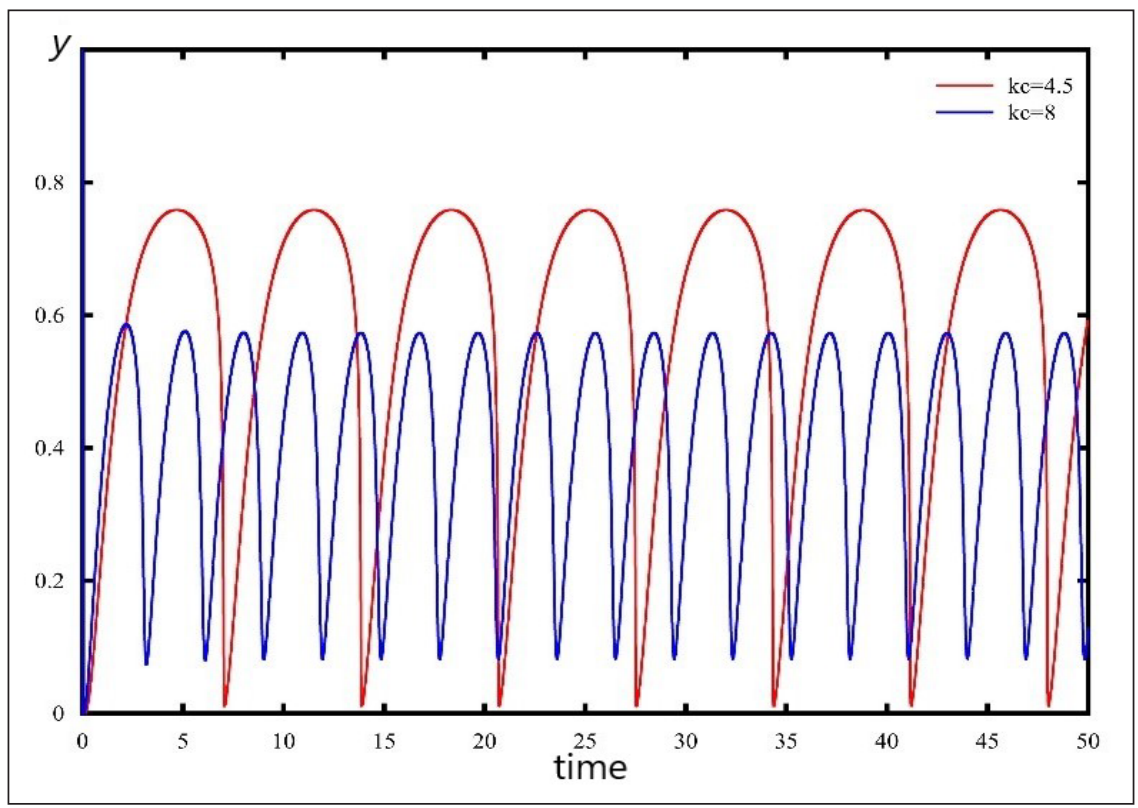

Figure 3. Time series plot at different values of $K_{C}$ 
analysis using two parameters which was the proportional control constant. $K_{C}$ and the dilution rate, $D$.

Effects of Varying the Proportional Control Constant. In this section, we analyse the dynamical behaviour of the system as the parameter $K_{C}$ changes. The term $K_{C}$ refers to a heat removal control that regulates the cooling process of water in this system. An increase in the value of $K_{C}$ cause a proportional increase in heat removal and hence lower the reactor temperature.

Figure 4 shows a bifurcation diagram at dilution rate $D=50$ when $K_{C}$ was varied from 0 to 12 . Red (Black, respectively) curves corresponded to stable (unstable, respectively) steady states. The green curves demonstrated the occurrence of stable limit cycle in this EBRS model. There also occurred several threshold points i.e., $H_{1}, H B_{1}, H B_{2}, S N$, and $\mathrm{H}_{2}$ which corresponded to first Hopf bifurcation, first homoclinic bifurcation, second homoclinic bifurcation, saddle-node bifurcation and second Hopf bifurcation respectively as $K_{C}$ increased to a higher value.

It was observed that at a very low $K_{C}$ e.g., $K_{C}=0$, the system had three steady state where the upper branch, $E_{3}$ and the lower branch $E_{1}$, of steady states were stable. They were separated by an unstable steady state in the middle branch, $E_{2}$. As $K_{C}$ increased, there was a dynamical changes occuring on the lower branch of stable steady state $E_{1}$ at critical point $H_{1}\left(K_{C}=1.813\right)$ corresponding to Hopf bifurcation. In this situation, the substrate

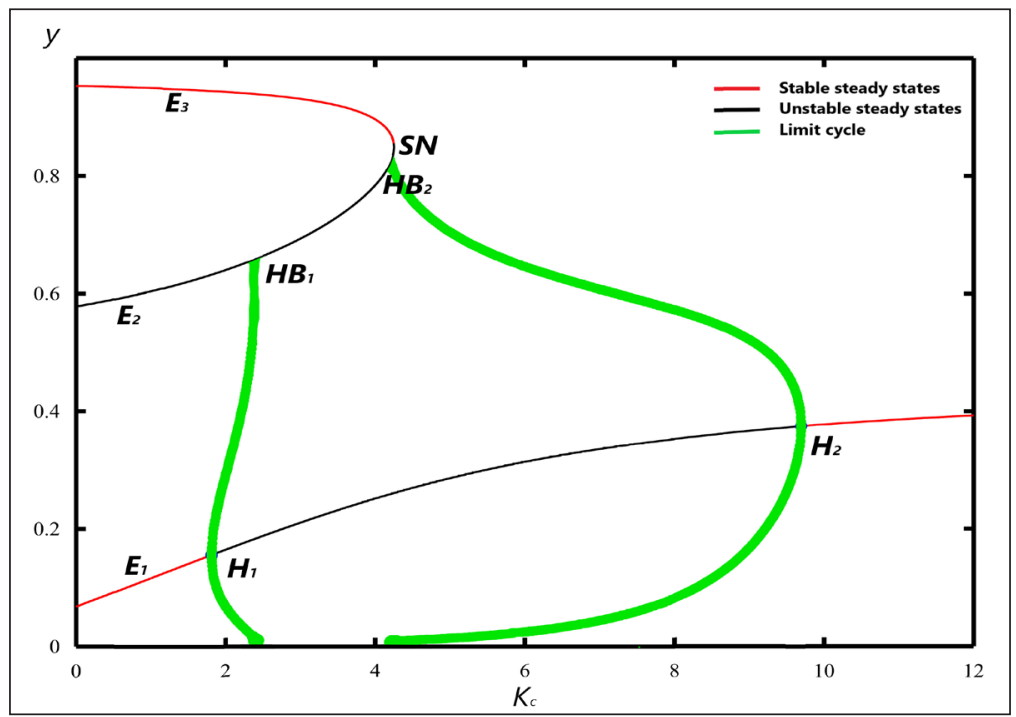

Figure 4. Bifurcation diagram for $K_{C}$ at $D=50$. Red curves correspond to stable steady states. The black curves correspond to the unstable steady state. The green curves demonstrate the occurrence of stable limit cycle. $E_{1}$ is the lower branch of steady state, $E_{2}$ is the middle branch of steady state and $E_{3}$ is the upper branch of steady state. $H_{1}, H B_{1}, H B_{2}, S N$, and $H_{2}$ correspond to first Hopf bifurcation, first homoclinic bifurcation, second homoclinic bifurcation, saddle-node bifurcation and second Hopf bifurcation respectively. 
concentration started to oscillate between certain minimum and maximum values. This observation corresponded to creation of a stable limit cycle. There was also a change in dynamical behaviour as previous bistability between two stable steady state in the region $K_{C}<H_{1}$ was replaced by a bistability between stable steady states and stable limit cycle in the region $H_{1}<K_{C}<H B_{1}$. As $K_{C}$ increased, the amplitude of oscillations in the stable limit cycle were getting bigger in size and consequently collided with the unstable saddle point in the middle branch $E_{2}$ at critical point $H B\left(K_{C}=2.396\right)$. This collision corresponded to a homoclinic bifurcation and resulting the disappearance of the previous stable limit cycle as $K_{C}$ was increased. As $K_{C}$ increased, there was another homoclinic bifurcation occurring at the critical point $H B\left(K_{C}=4.203\right)$. There was a new dynamical behaviour observed in the region $H B_{1}<K_{C}<H B_{2}$ as the previous bistability between stable limit cycle and stable steady state was replaced by single steady states. All trajectories would converge to a stable steady state in upper branch $E_{3}$. As $K_{C}$ increased pass the $H B_{2}$, we observed another appearance of stable limit cycle. This limit cycle resulted from the second Hopf bifurcation occurring at critical point $H_{2}\left(K_{C}=9.694\right)$ in the lower branch, $E_{1}$. Meanwhile, in the upper branch $E_{3}$ there was another critical point at $\operatorname{SN}\left(K_{C}=4.251\right)$ corresponding to a saddle-node bifurcation. This saddle-node bifurcation resulted from the collision between stable steady state in upper branch, $E_{3}$ and unstable steady state in middle branch, $E_{2}$. We also observed the dynamical behaviour changes in the region $\mathrm{HB}_{2}$ $<K_{C}<S N$ as the bistability occurred between stable steady state and stable limit cycle. Another changes in dynamical behaviour was observed as the previous bistability was changed to single steady state in region $\mathrm{K}_{\mathrm{C}}>\mathrm{H}_{2}$.

Effect of Varying the Dilution Rate. In this analysis, we investigated the influence of the dilution rate $D$ on the outcomes of the system. Since the choice of the parameter $K_{C}$ value was quite arbitrary, we set the outcome of the model for four distinct values of $K_{C}$ so as to capture the overall dynamic of the model.

When the dilution rate, $D$ increased, we observed the occurrence of different threshold values that would determine the dynamical behaviour of EBRS model. As we can see in Figure 5, there was a critical point occurring at $H(D=40.59)$ which is corresponded to Hopf bifurcation. We observed as $D<H$, the system would be in stable steady state. On the other hand, as $D>H$, the stable steady state turned into unstable steady state, with corresponded to the creation of stable limit cycle. In this situation, the substrate concentration started to oscillate between certain minimum and maximum values. The stable limit cycle terminated at critical point $H B(D=49.3)$ corresponding to homoclinic bifurcation. The homoclinic bifurcation occurred as a result from the collision of stable limit cycle with the saddle point. There was also another critical point occurring at $S N(D=48.52)$ corresponding 


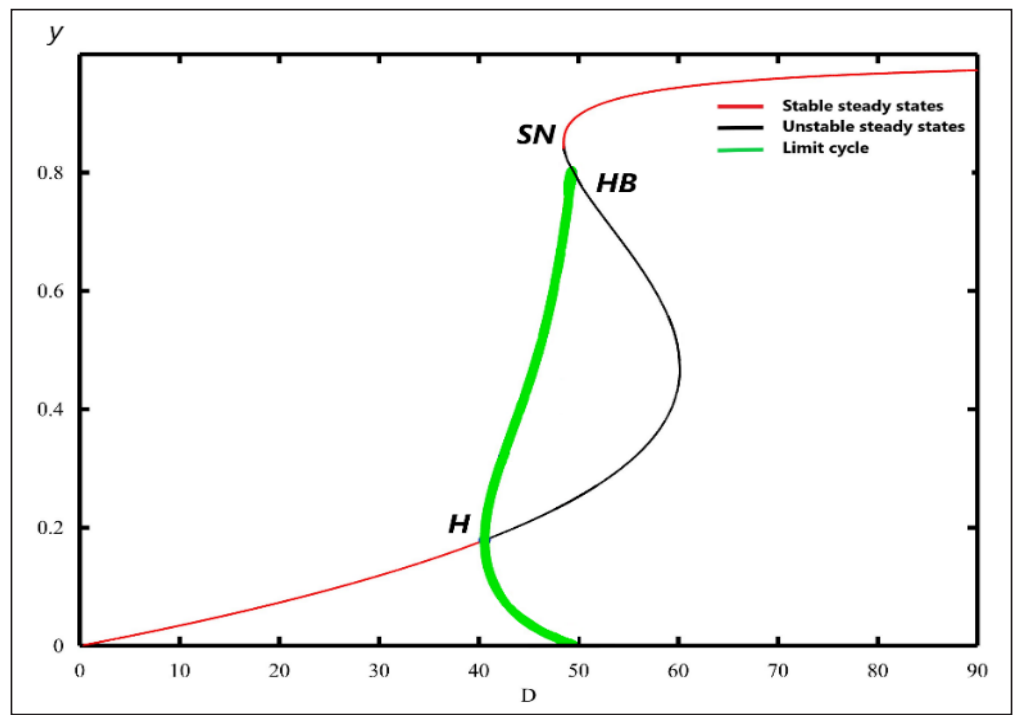

Figure 5. Bifurcation diagram for $D$ at $K_{C}=4$. Red curves correspond to stable steady states. The black curves correspond to the unstable steady state. The green curves demonstrate the occurrence of stable limit cycle. $H$, $H B$ and $S N$ correspond to Hopf bifurcation, homoclinic bifurcation, and saddle-node bifurcation respectively.

to the saddle node bifurcation as the stable steady state in the upper region collided with unstable steady state in the middle region. There existed a region of bistability between stable steady state and stable limit cycle in the region $S N<D<H B$.

\section{Codimension-Two Bifurcation Analysis}

Throughout this section, a codimension-two bifurcation analysis was conducted to continue the detected bifurcations through two parameter spaces and examined the boundary of the stable region. We would investigate the joint effects of proportional control constant, $K_{C}$ and dilution rate, $D$ on the dynamics of the system. To illustrate the effect of increasing the proportional control constant $K_{C}$ on the dynamics of the system as dilution rate $D$ varies, several bifurcation diagrams with different $K_{C}$ values were plotted in Figure 6.

Figure 6 a shows the bifurcation diagram with respect to parameter $D$ and it was plotted at $K_{C}=2$. The were three critical points observe in the bifurcation diagram corresponding to Hopf bifurcation, $H$ at $D=48.66$, homoclinic bifurcation, $H B$ at $D=51.61$ and saddle node bifurcation, $S N$ at $D=38.29$. There were two regions of bistability observed. The first bistability occurred in the region $S N<D<H$ between stable steady state in the higher concentration of $y$ and the stable steady state in the lower concentration of $y$ (red curve). Second bistability occurred in the region $H<D<H B$ between the stable steady state in the higher substrate concentration of $y$ and the stable limit cycle emerging from the Hopf bifurcation in lower substrate concentration of $y$. Figure $6 \mathrm{~b}$ shows the bifurcation diagram with respect to parameter $D$ as we increased the value of $K_{C}=5$. We observed a dynamical 


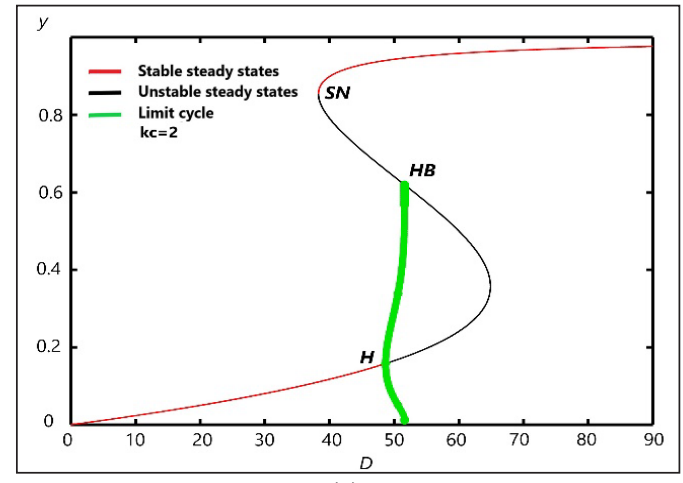

(a)

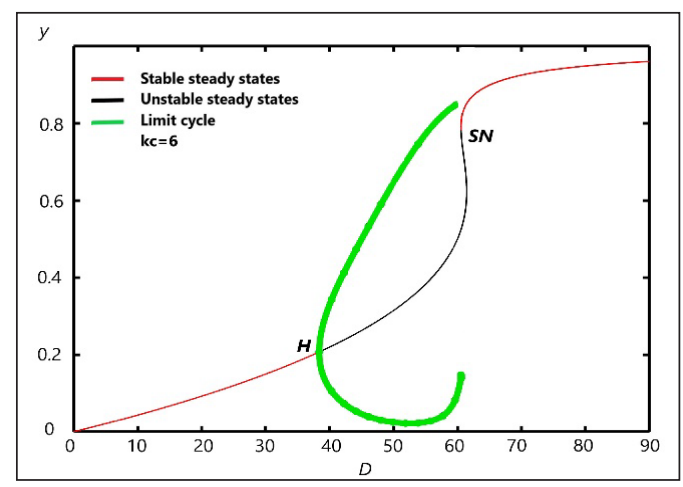

(c)

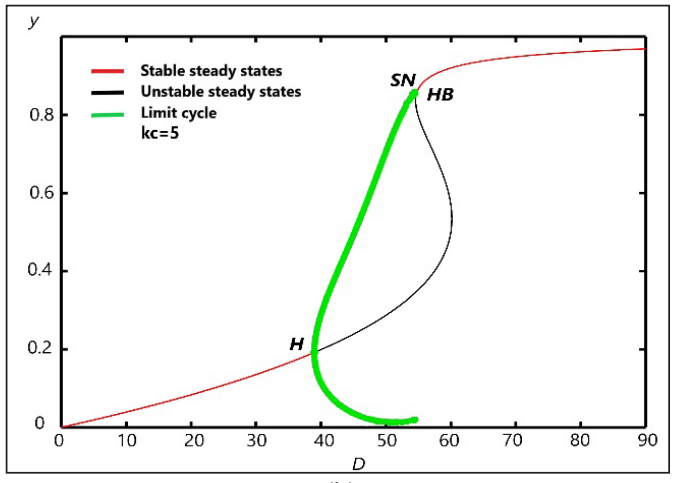

(b)

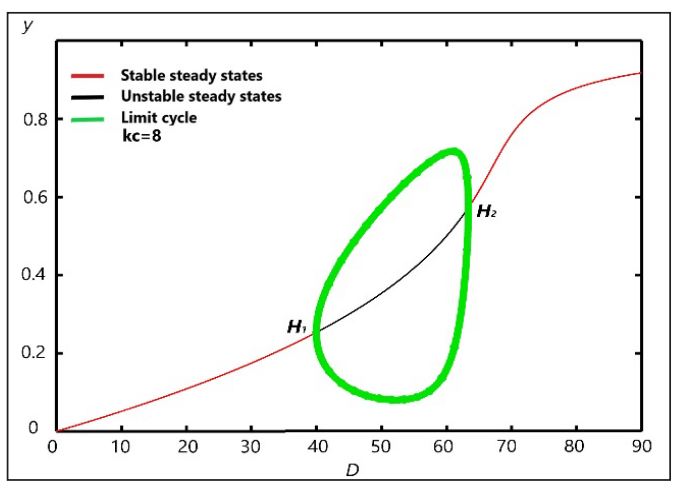

(d)

Figure 6. The effect of different $K_{C}$ on the dynamics of the system as $D$ increases. Red curves correspond to stable steady states. The black curves correspond to the unstable steady state. The green curves demonstrate the occurrence of stable limit cycle. $H, H_{1}, H_{2}, H B$, and $S N$ correspond to Hopf bifurcation, first Hopf bifurcation, second Hopf bifurcation, homoclinic bifurcation, and saddle-node bifurcation respectively.

behaviour had changed compared to previous observation. There were three bifurcation observed in the bifurcation diagram corresponding to Hopf bifurcation, $H$ occurring at $D=$ 38.99 , the homoclinic bifurcation, $H B$ occurring at $D=54.51$ and saddle node bifurcation, $S N$ occurring at $D=54.58$. We also observed the 'S' shape of the curve seemed to 'unfold' and this situation led to the previous bistabililty between two stable steady state in Figure $6 \mathrm{a}$ disappeared. Moreover, the bistability region between the stable steady state in the higher substrate concentration of $y$ and the stable limit dcycle emerged from the Hopf bifurcation in lower substrate concentration of $y$ shrinking to a very small region in the region $H B$ $<D<S N$. Figure $6 \mathrm{c}$ shows the bifurcation diagram with respect to parameter $D$ as we increased the value of $K_{C}=6$. We observed the disappearance of homoclinic bifurcation in the previous observation. Two bifurcations were observed in the bifurcation diagram corresponding to Hopf bifurcation, $H$ at $D=38.38$ and saddle node, $S N$ bifurcation at $D=$ 60.55. The ' $\mathrm{S}$ ' shape of the cure became flaten with no more bistability phenomen occurring in the system. Figure $6 \mathrm{~d}$ shows the bifurcation diagram with respect to parameter $D$ as we 
increased the value of $K_{C}=8$. Two Hopf bifurcation emerged from the system with the first Hopf bifurcation, $H_{1}$ occurring at $D=39.98$ and second Hopf bifurcation, $H_{2}$ occurring at $D=63.36$. This two Hopf bifurcation were connected by a stable limit cycle bifurcation from each critical point $H_{1}$ and $H_{2}$. There was no bistability phenomenon occurring and the substrate concentration increased as the dilution rate increased.

There are three salient features we discovered from increasing the proportional control constant $K_{C}$ on the dynamics of the system as dilution rate $D$ varied. The first one was the disappearance of the bistability phenomenon; the bistability observed disappearing at higher $K_{C}$ (Figure $6 \mathrm{c}$ to d). The second observation was the disappearance of the saddle node and homoclinic bifurcations; the ' $\mathrm{S}$ ' shape of the equilibrium curve in lower $K_{C}$ seemed to 'unfold' with increasing of $K_{C}$ (Figure 6c). The third observation was the occurrence of two Hopf bifurcations. At high values of $K_{C}$, limit cycles which occurred was connected by two Hopf bifurcation points (Figure 6d). These three features are best described using a two-parameter bifurcation diagram.

Figure 7 shows a codimension-two bifurcation diagram as parameters $D$ and $K_{C}$ vary. The formation of Bogdanov-Takens point (BT) at $K_{C}=6.25035$ and $D=62.048275$ marked the meeting of the saddle node bifurcation and the Hopf bifurcations. The parameter space was divided into five regions by the Hopf, saddle node and homoclinic bifurcation curves. These regions (labelled I, II, III, IV and V) corresponded to distinct stable steady states of the system. Region I corresponded to the single stable steady state, region II corresponded

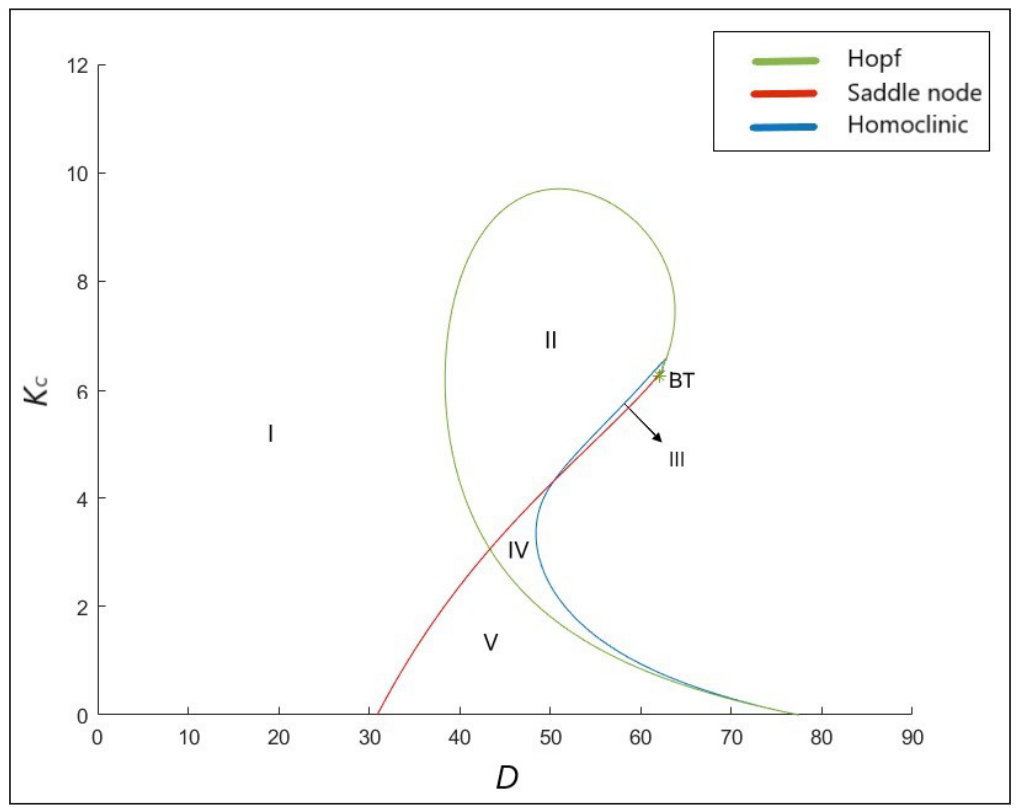

Figure 7. Codimension-two parameter bifurcation diagram. Green curve refers to Hopf bifurcation, red curve refers saddle node bifurcation and blue curve refers to homoclinic bifurcation. BT is the Bogdanov-Takens bifurcation point. 
to the stable limit cycle, regions III and IV corresponded to the bistability between the steady states and limit cycle and region $\mathrm{V}$ corresponded to the bistability between two stable steady states.

\section{DISCUSSION AND CONCLUSION}

There is one limitation in our result. In order to analysis the stability of the solution in the EBRS model, it is very difficult to solve the model analytically since the model involves large set of parameters and highly nonlinear. We need to rely on the computer software such as MAPLE ${ }^{\circledR}$ and XPPAUT to compute the solutions of the model and determine their stability. Hence, all the results we present are computed numerically.

In a biocatalytic reaction, it is crucial to understand the dynamics of the reaction in CSTR by identifying the critical transitions between the possible dynamical regimes. Different parameters such as temperature and dilution rate are vital components in achieving high efficiency (productivity) of the reactor. Our results have shown that when the value $K_{C}$ is high (e.g. $K_{C}>10$ ) the system will achieve a stable steady state (Figure 7) regardless of how fast the dilution rate is. For higher values of $K_{C}$, more heat is removed from the system, lowering the reactor temperature level and providing optimal conditions for the biocatalytic reaction to operate. When the value of $K_{C}$ is low (e.g., $K_{C}=2$ ), minimal heat is removed from the system and the accumulated heat affects the biocatalytic reaction. At certain dilution rates $D$, the system exhibits different dynamics: (i) a stable steady state (e.g., $D=20$ ), (ii) bistability between two stable steady states (e.g., $D=40$ ) and (iii) bistability between the stable steady state and stable limit cycle (e.g., $D=50)$.

Controlling the dilution rate is also important in the CSTR operations. A reaction at higher and lower dilution rates provides better stability for the CSTR operations. For a higher dilution rate, the substrate concentration is high (Figure 6). This is due to the shorter duration that the substrate spends in the reactor. The enzymes are not fully utilised since the inflow and outflow of the reactant and product are at a higher rate. Lowering the dilution rate will result in a lower substrate concentration because the enzyme has more time to catalyse the reaction.

The finding of this study is also consistent with the result in Radzi and Uzir (2009). The dynamical behaviours are qualitatively the same. However, this study extends the previous knowledge in Radzi and Uzir (2009) by discovering the homoclinic bifurcation as a result of collision between stable limit cycle and saddle point in unstable steady state. Moreover, we have detected the region of bistability in which Radzi and Uzir (2009) missed. We also extending the bifurcation result into codimension-two bifurcations diagram and reveal more dynamical behaviours lies within the exothermic biocatalytic reaction model. 
To conclude the discussion, the outcomes of the system depend on the joint influence of proportional control constant, $K_{C}$ and the dilution rate, $D$. This study has provided a detailed analysis of the dynamical behaviours of an exothermic biocatalytic reaction as some chemically relevant parameters change. Using bifurcation analysis, the role of proportional control constant $K_{C}$ and the dilution rate $D$ in determining the outcomes of this chemical system is understood. For future research, other parameters could be taken into consideration such as enzyme concentration. Additionally, a specific inhibition model, such as competitive inhibition or uncompetitive inhibition, can be formulated and the results can be compared with this model.

\section{ACKNOWLEDGEMENTS}

Thanks to the School of Mathematical Sciences and the Universiti Sains Malaysia for the support. Mohd Hafiz Mohd is supported by Universiti Sains Malaysia (USM) Fundamental Research Grant Scheme (FRGS) No. 203/PMATHS/6711645.

\section{REFERENCES}

Aris, R., \& Amundson, N. R. (1958a). An analysis of chemical reactor stability and control-I: The possibility of local control, with perfect or imperfect control mechanisms. Chemical Engineering Science, 7(3), 121-131. doi: https://doi.org/10.1016/0009-2509(58)80019-6

Aris, R., \& Amundson, N. R. (1958b). An analysis of chemical reactor stability and control-II: The evolution of proportional control. Chemical Engineering Science, 7(3), 132-147. doi: https://doi.org/10.1016/00092509(58)80020-2

Bommarius, A. S., \& Riebel, B. R. (2004). Biocatalysis, fundamentals and applications. Weinheim, Germany: John Wiley \& Sons.

Choi, J. M., Han, S. S., \& Kim, H. S. (2015). Industrial applications of enzyme biocatalysis: Current status and future aspects. Biotechnology Advances, 33(7), 1443-1454. doi: https://doi.org/10.1016/j. biotechadv.2015.02.014

De Gooijer, C. D., Bakker, W. A. M., Beeftink, H. H., \& Tramper, J. (1996). Bioreactors in series: An overview of design procedures and practical applications. Enzyme and Microbial Technology, 18(3), 202-219. doi: https://doi.org/10.1016/0141-0229(95)00090-9

Devasena, T. (2010). Enzymology. New Delhi, India: Oxford University Press.

Guan, D., Li, P., Zhang, Q., Zhang, W., Zhang, D., \& Jiang, J. (2011). An ultra-sensitive monoclonal antibodybased competitive enzyme immunoassay for aflatoxin M1 in milk and infant milk products. Food Chemistry, 125(4), 1359-1364. doi: https://doi.org/10.1016/j.foodchem.2010.10.006

Guengerich, F. P. (2017). Mechanisms of enzyme catalysis and inhibition. In D. L. Eaton (Ed.), Comprehensive toxicology (3rd Ed., Vol. 10-15). London, UK: Elsevier. doi: https://doi.org/10.1016/B978-0-12-801238$3.10922-5$ 
Hu, K., Huang, X., Jiang, Y., Fang, W., \& Yang, X. (2010). Monoclonal antibody based enzyme-linked immunosorbent assay for the specific detection of ciprofloxacin and enrofloxacin residues in fishery products. Aquaculture, 310(1-2), 8-12. doi: https://doi.org/10.1016/j.aquaculture.2010.08.008

Jestin, J. L., \& Kaminski, P. A. (2004). Directed enzyme evolution and selections for catalysis based on product formation. Journal of Biotechnology, 113(1-3), 85-103. doi: https://doi.org/10.1016/j.jbiotec.2004.03.032

Lindeque, R. M., \& Woodley, J. M. (2019). Reactor selection for effective continuous biocatalytic production of pharmaceuticals. Catalysts, 9(3), 1-17. doi: https://doi.org/10.3390/catal9030262

Mazzei, R., Giorno, L., Piacentini, E., Mazzuca, S., \& Drioli, E. (2009). Kinetic study of a biocatalytic membrane reactor containing immobilized $\beta$-glucosidase for the hydrolysis of oleuropein. Journal of Membrane Science, 339(1-2), 215-223. doi: https://doi.org/10.1016/j.memsci.2009.04.053

Petkevicius, L., \& Baronas, R. (2017, October 8-12). Numerical simulation and analysis of enzyme-catalysed substrate conversion in a microbioreactor. In SIMUL 2017: The Ninth International Conference on Advances in System Simulation (pp. 1-6). Athens, Greece.

Petrucci, R. H., Harwood, W. S., \& Herring, F. G. (2002). General chemistry: Principles and modern applications (Vol. 1). Upper Saddle River, NJ: Prentice Hall.

Radzi, M. R. M., \& Uzir, M. H. (2009). Stability study of an exothermic biocatalytic reaction and its application in bioprocess systems. Pertanika Journal of Science and Technology, 17(1), 95-115.

Sekhon, J. K., Rosentrater, K. A., Jung, S., \& Wang, T. (2018). Effect of co-products of enzyme-assisted aqueous extraction of soybeans, enzymes, and surfactant on oil recovery from integrated corn-soy fermentation. Industrial Crops and Products, 121(December 2017), 441-451. doi: https://doi.org/10.1016/j. indcrop.2018.05.033

Tufvesson, P., Fu, W., Jensen, J. S., \& Woodley, J. M. (2010). Process considerations for the scale-up and implementation of biocatalysis. Food and Bioproducts Processing, 88(1), 3-11. doi: https://doi. org/10.1016/j.fbp.2010.01.003

Uhr, L., Buchholz, T., Homann, T., Huschek, G., \& Rawel, H. M. (2014). Targeted proteomics-based analysis of technical enzymes from fungal origin in baked products. Journal of Cereal Science, 60(2), 440-447. doi: https://doi.org/10.1016/j.jcs.2014.04.007

Zhao, Y. M., Wang, L. H., Luo, S. F., Wang, Q. Q., Moaddel, R., Zhang, T. T., \& Jiang, Z. J. (2018). Magnetic beads-based neuraminidase enzyme microreactor as a drug discovery tool for screening inhibitors from compound libraries and fishing ligands from natural products. Journal of Chromatography A, 1568, 123130. doi: https://doi.org/10.1016/j.chroma.2018.07.031 